Mon. Not. R. Astron. Soc. 000,15(2015) Printed 8 November $2018 \quad$ (MN LTEX style file v2.2)

\title{
A search for the presence of magnetic fields in the two Supergiant Fast X-ray Transients IGR J08408-4503 and IGR J11215-5952
}

\author{
S. Hubrig ${ }^{1 \star}$, L. Sidoli ${ }^{2}$, K. Postnov ${ }^{3}$, M. Schöller ${ }^{4}$, A. F. Kholtygin ${ }^{5}$, S. P. Järvinen ${ }^{1}$, \\ P. Steinbrunner 6 \\ ${ }^{1}$ Leibniz-Institut für Astrophysik Potsdam (AIP), An der Sternwarte 16, 14482 Potsdam, Germany \\ ${ }^{2}$ INAF, Istituto di Astrofisica Spaziale e Fisica Cosmica, Via E. Bassini 15, 20133 Milano, Italy \\ ${ }^{3}$ Sternberg Astronomical Institute, Moscow M.V. Lomonosov State University, 119234 Moscow, Russia \\ ${ }^{4}$ European Southern Observatory, Karl-Schwarzschild-Str. 2, 85748 Garching, Germany \\ ${ }^{5}$ Saint-Petersburg State University, Universitetskij pr. 28, Saint-Petersburg 198504, Russia \\ ${ }^{6}$ Freie Universität Berlin, Kaiserswerther Str. 16-18, 14195 Berlin, Germany
}

Accepted Received; in original form

\begin{abstract}
A significant fraction of high-mass X-ray binaries are supergiant fast X-ray transients (SFXTs). The prime model for the physics governing their X-ray behaviour suggests that the winds of donor OB supergiants are magnetized. To investigate if magnetic fields are indeed present in the optical counterparts of such systems, we acquired low-resolution spectropolarimetric observations of the two optically brightest SFXTs, IGR J08408-4503 and IGR J11215-5952 with the ESO FORS 2 instrument during two different observing runs. No field detection at a significance level of $3 \sigma$ was achieved for IGR J08408-4503. For IGR J11215-5952, we obtain $3.2 \sigma$ and $3.8 \sigma$ detections $\left(\left\langle B_{\mathrm{z}}\right\rangle_{\mathrm{hydr}}=-978 \pm 308 \mathrm{G}\right.$ and $\left\langle B_{\mathrm{z}}\right\rangle_{\mathrm{hydr}}=416 \pm 110 \mathrm{G}$ ) on two different nights in 2016 . These results indicate that the model involving the interaction of a magnetized stellar wind with the neutron star magnetosphere can indeed be considered to characterize the behaviour of SFXTs. We detected long-term spectral variability in IGR J11215-5952, while for IGR J08408-4503 we find an indication of the presence of short-term variability on a time scale of minutes.
\end{abstract}

Key words: stars: supergiants — stars: individual: IGR J08408-4503, IGR J11215-5952 — stars: magnetic fields - X-rays: stars — stars: binaries

\section{INTRODUCTION}

Among the bright $\mathrm{X}$-ray sources in the sky, a significant number contain a compact object (either a neutron star or a black hole) accreting from the wind of a companion star with a mass above $10 M_{\odot}$ (Liu et al. 2006). Such systems are called high-mass X-ray binaries (HMXBs). They are young (several dozen million years old) and can be formed when one of the initial binary members loses a significant part of its mass through stellar wind or mass transfer before a first supernova explosion occurs (van den Heuvel \& Heise 1972). Studies of different types of HMXBs are of special interest to obtain reliable predictions about the populations of relativistic binaries such as double degenerate binaries, which are considered to be gravitational wave progenitors. Indeed, just a few weeks ago, scientists have directly detected gravitational waves from the spectacular collision of two neutron stars (e.g., Cowperthwaite et al. 2017). Furthermore, high-mass X-ray binaries are fundamental for

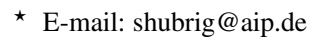

studying stellar evolution, nucleosynthesis, structure and evolution of galaxies, and accretion processes.

Supergiant Fast X-ray Transients (SFXTs) are a subclass of HMXBs associated with early-type supergiant companions, and characterized by sporadic, short and bright Xray flares reaching peak luminosities of $10^{36}-10^{37} \mathrm{erg} \mathrm{s}^{-1}$ and typical energies released in bright flares of about $10^{38}-10^{40}$ erg (see the review of Sidoli 2017 for more details). Their X-ray spectra in outburst are very similar to accreting pulsars in HMXBs. In fact, half of them have measured neutron star spin periods similar to those observed from persistent HMXBs (Shakura et al. 2015; ; Martinez-Nunez et al. 2017).

The physical mechanism driving their transient behavior, probably related to the accretion of matter from the supergiant wind by the compact object, has been discussed by several authors and is still a matter of debate. The prime model for the existence of SFXTs invokes their different wind properties and magnetic field strengths, which lead to distinctive accretion regimes (Shakura et al. 2012, 2014; Shakura \& Postnov 2017). The SFXTs' behaviour can be explained by sporadic capture of magnetized stellar wind. The effect of the magnetic field carried by the stellar wind is twofold: first, it 
Table 1. Logbook of the FORS 2 polarimetric observations of IGR J08408-4503 and IGR J11215-5952, including the modified Julian date of mid-exposure, followed by the achieved signal-to-noise ratio in the Stokes $I$ spectra around $5200 \AA$, and the measurements of the mean longitudinal magnetic field using the Monte Carlo bootstrapping test, for the hydrogen lines and for all lines. In the last columns, we present the results of our measurements using the null spectra for the set of all lines, and the orbital phase (see text). All quoted errors are $1 \sigma$ uncertainties.

\begin{tabular}{ccccrc}
\hline \hline $\begin{array}{c}\text { MJD } \\
57000+\end{array}$ & $\begin{array}{c}\text { SNR } \\
\lambda 5200\end{array}$ & $\begin{array}{c}\left\langle B_{\mathrm{z}}\right\rangle_{\text {hydr }} \\
{[\mathrm{G}]}\end{array}$ & $\begin{array}{c}\left\langle B_{\mathrm{z}}\right\rangle_{\text {all }} \\
{[\mathrm{G}]}\end{array}$ & \multicolumn{1}{c}{$\begin{array}{c}\left\langle B_{\mathrm{z}}\right\rangle_{\mathrm{N}} \\
{[\mathrm{G}]}\end{array}$} & $\varphi_{\text {orb }}$ \\
\hline \multicolumn{7}{c}{ IGR J08408-4503 } \\
\hline 727.3424 & 2730 & $21 \pm 132$ & $162 \pm 114$ & $87 \pm 135$ & 0.080 \\
736.2242 & 3391 & $-184 \pm 97$ & $-29 \pm 59$ & $-37 \pm 62$ & 0.011 \\
745.2449 & 1844 & $269 \pm 158$ & $131 \pm 100$ & $-93 \pm 82$ & 0.956 \\
747.2578 & 3401 & $-141 \pm 94$ & $18 \pm 67$ & $-39 \pm 63$ & 0.167 \\
\hline \multicolumn{7}{c}{ IGR J11215-5952 } \\
\hline 522.0581 & 1012 & $-978 \pm 308$ & $-646 \pm 317$ & $0 \pm 356$ & 0.958 \\
528.0357 & 1926 & $406 \pm 156$ & $222 \pm 93$ & $-44 \pm 93$ & 0.995 \\
738.2863 & 2844 & $416 \pm 110$ & $208 \pm 62$ & $31 \pm 67$ & 0.272 \\
764.2995 & 1957 & $-96 \pm 145$ & $116 \pm 73$ & $-143 \pm 88$ & 0.430 \\
793.3257 & 2849 & $241 \pm 113$ & $133 \pm 55$ & $67 \pm 48$ & 0.606 \\
\hline
\end{tabular}

may trigger rapid mass entry to the magnetosphere via magnetic reconnection in the magnetopause (a phenomenon that is well known in the dayside of the Earth magnetosphere), and secondly, the magnetized parts of the wind (magnetized clumps with a tangent magnetic field) have a lower velocity than the non-magnetized parts or the parts carrying the radial field (Shakura et al. 2014). The model predicts that a magnetized clump of stellar wind with a magnetic field strength of a few tens of Gauss triggers sporadic reconnection, allows accretion, and results in an X-ray flare. Since, typically, the orbital separation between the neutron star and the supergiant is a few $R_{*}$ of the latter, the expected required magnetic field on the stellar surface should be of the order of $100-1000 \mathrm{G}$.

To investigate the magnetic nature of the optical counterparts in these systems, we recently observed the two optically brightest SFXTs, IGR J08408-4503 $\left(m_{V}=7.6\right)$ and IGR J11215-5952 $\left(m_{V}=10.0\right)$, using the FOcal Reducer low dispersion Spectrograph (FORS 2; Appenzeller et al. 1998) mounted on the $8 \mathrm{~m}$ Antu telescope of the VLT. The optical counterpart of the system IGR J08408-4503 is the O-type supergiant star HD 74194 with spectral classification O8.6 Ib-II(f)p (Sota et al. 2014). The orbital solution $\left(P_{\text {orb }}=9.5436 \pm 0.002 \mathrm{~d}, e=0.63 \pm 0.03\right)$ was for the first time determined by Gamen et al. (2015). This work also indicated that the equivalent width of the $\mathrm{H} \alpha$ line is not modulated entirely with the orbital period, but seemed to vary with a superorbital pe$\operatorname{riod}(P=285 \pm 10 \mathrm{~d})$ nearly 30 times longer than the orbital one. This orbital solution also supported the existence of a correlation between high-energy outbursts and periastron passages.

The astrophysical parameters of the optical counterpart of the system IGR J11215-5952, the B0.5 Ia supergiant HD 306414, were recently studied by Lorenzo et al. (2014), who suggested that this supergiant of about $30 M_{\odot}$ has just completed $\mathrm{H}$ core burning, showing only slight enhancement of He and N. No clear orbital variability, neither in radial velocities nor in the photometric light curve, was detected. X-ray outbursts occurring every $164.6 \mathrm{~d}$ (Sidoli et al. 2007; Romano et al. 2009) strongly suggest that this recurrence timescale is the orbital period. In this case, the outbursts

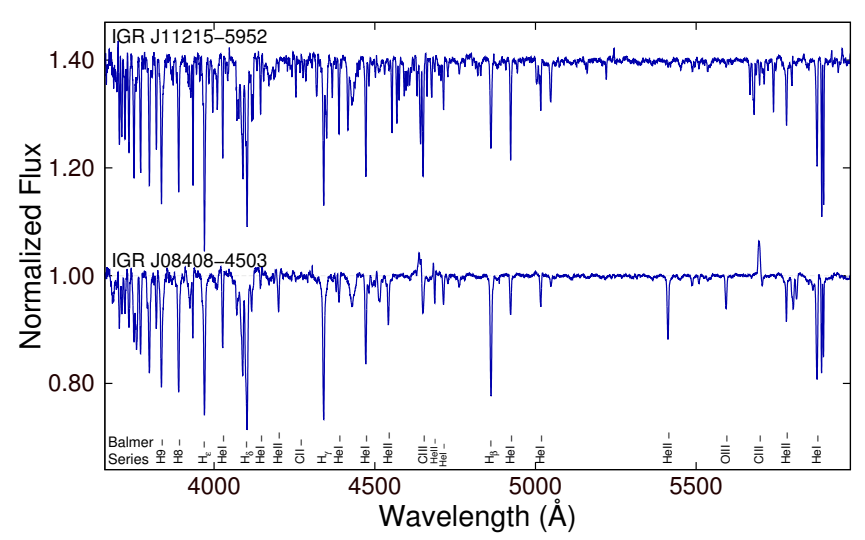

Figure 1. Normalised FORS 2 Stokes $I$ spectra of IGR J08408-4503 and IGR J11215-5952. Well known spectral lines are indicated at the bottom. The spectrum of IGR J11215-5952 was vertically offset by 0.4 for clarity.

are thought to be produced near the periastron passage, although the real orbital phase where they occur is unknown.

High-resolution optical spectroscopy with the FEROS instrument mounted on the ESO/MPG 2.2 m telescope on La Silla (Chile) suggested a high orbital eccentricity and the presence of pulsations causing the measured radial velocity curve to deviate significantly from that expected from Keplerian motion (Lorenzo et al. 2014). Since, according to the model, supergiant magnetic fields can play a major role for the generation of outbursts, we carried out the first search ever for magnetic fields in the two brightest SFXTs accessible with the VLT. In the following, we present the results of our magnetic field measurements and discuss the detected spectral variability.

\section{OBSERVATIONS AND MAGNETIC FIELD MEASUREMENTS}

FORS 2 spectropolarimetric observations of both systems were obtained from 2016 May 14 to 2017 February 9. The FORS 2 multimode instrument is equipped with polarisation analysing optics comprising super-achromatic half-wave and quarter-wave phase retarder plates, and a Wollaston prism with a beam divergence of $22^{\prime \prime}$ in standard resolution mode. We used the GRISM 600B and the narrowest available slit width of 0 .' $^{\prime} 4$ to obtain a spectral resolving power of $R \sim 2000$. The observed spectral range from 3250 to $6215 \AA$ includes all Balmer lines, apart from $\mathrm{H} \alpha$, and numerous helium lines. For the observations, we used a non-standard readout mode with low gain $(200 \mathrm{kHz}, 1 \times 1$, low $)$, which provides a broader dynamic range, hence allowed us to reach a higher signal-to-noise ratio (SNR) in the individual spectra. The spectral appearance of IGR J08408-4503 and IGR J11215-5952 in the FORS 2 spectra is presented in Fig. 1 A description of the assessment of the presence of a longitudinal magnetic field using FORS $1 / 2$ spectropolarimetric observations was presented in our previous work (e.g. Hubrig et al. 2004a b, and references therein).

Rectification of the $V / I$ spectra was performed in the way described by Hubrig, Schöller, \& Kholtygin (2014). Null profiles, $N$, are calculated as pairwise differences from all available $V$ profiles so that the real polarisation signal should cancel out. From these, $3 \sigma$-outliers are identified and used to clip the $V$ profiles. This removes spurious signals, which mostly come from cosmic rays, and also reduces the noise. A full description of the updated data reduc- 

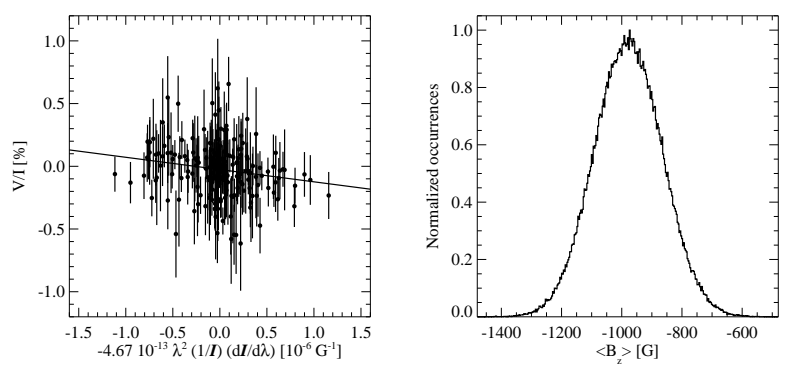

Figure 2. Left panel: Linear fit to Stokes $V$ spectrum obtained for the FORS 2 observation of IGR J11215-5952 on MJD 57522.0581. Right panel: Distribution of the longitudinal magnetic field values $P\left(\left\langle B_{\mathrm{Z}}\right\rangle\right)$, which were obtained via bootstrapping. From this distribution follows the most likely value for the longitudinal magnetic field $\left\langle B_{\mathrm{z}}\right\rangle_{\mathrm{hydr}}=-978 \pm 308 \mathrm{G}$.

tion and analysis will be presented in a separate paper (Schöller et al., in preparation, see also Hubrig. Schöller. \& Kholtygin 2014). The mean longitudinal magnetic field, $\left\langle B_{z}\right\rangle$, is measured on the rectified and clipped spectra based on the relation following the method suggested by Angel \& Landstreet (1970)

$\frac{V}{I}=-\frac{g_{\mathrm{eff}} e \lambda^{2}}{4 \pi m_{\mathrm{e}} c^{2}} \frac{1}{I} \frac{\mathrm{d} I}{\mathrm{~d} \lambda}\left\langle B_{\mathrm{z}}\right\rangle$,

where $V$ is the Stokes parameter that measures the circular polarization, $I$ is the intensity in the unpolarized spectrum, $g_{\text {eff }}$ is the effective Landé factor, $e$ is the electron charge, $\lambda$ is the wavelength, $m_{\mathrm{e}}$ is the electron mass, $c$ is the speed of light, $\mathrm{d} I / \mathrm{d} \lambda$ is the wavelength derivative of Stokes $I$, and $\left\langle B_{z}\right\rangle$ is the mean longitudinal (line-ofsight) magnetic field.

The longitudinal magnetic field was measured in two ways: using the entire spectrum including all available lines, among them 13 strong He lines, or using exclusively hydrogen lines, 13 in total. Furthermore, we have carried out Monte Carlo bootstrapping tests. These are most often applied with the purpose of deriving robust estimates of standard errors (e.g. Steffen et al. 2014). The measurement uncertainties obtained before and after the Monte Carlo bootstrapping tests were found to be in close agreement, indicating the absence of reduction flaws. The results of our magnetic field measurements, those for the entire spectrum or only the hydrogen lines are presented in Table 1 The orbital phases of IGR J08408-4503 were calculated relative to a zero phase corresponding to the periastron passage at $T_{\text {per }}=2454654.04$ (Gamen et al. 2015). The same zero phase as in the work of Lorenzo et al. (2014) corresponding to the date of the first FEROS observation was adopted for IGR J11215-5952. The X-ray outbursts occurred at orbital phases close to 0.4, following Lorenzo et al. (2014).

The magnetic field of IGR J08408-4503 was measured on four nights in 2016, but no detection at a significance level of $3 \sigma$ was achieved in any of the measurements. The magnetic field, if present, would likely be rather weak or variable. The highest value for the longitudinal magnetic field, $\left\langle B_{z}\right\rangle_{\mathrm{hydr}}=-184 \pm 97 \mathrm{G}$ at a significance level of $1.9 \sigma$ was measured in the observation obtained on 2016 December 14. For IGR J11215-5952, we obtain $3.2 \sigma\left(\left\langle B_{\mathrm{z}}\right\rangle_{\mathrm{hydr}}=-978 \pm 308 \mathrm{G}\right)$ and $3.8 \sigma\left(\left\langle B_{\mathrm{z}}\right\rangle_{\mathrm{hydr}}=416 \pm 110 \mathrm{G}\right)$ detections on two observing nights, on 2016 May 14 and 2016 December 16, respectively. In Figs 2 and 3 we show linear regressions in the plots $V / I$ against $-4.6710^{-13} \lambda^{2}(1 / I)(\mathrm{d} I / \mathrm{d} \lambda)$ together with the results of the Monte Carlo bootstrapping tests. No detection was achieved in the diagnostic $N$ spectra, indicating the absence
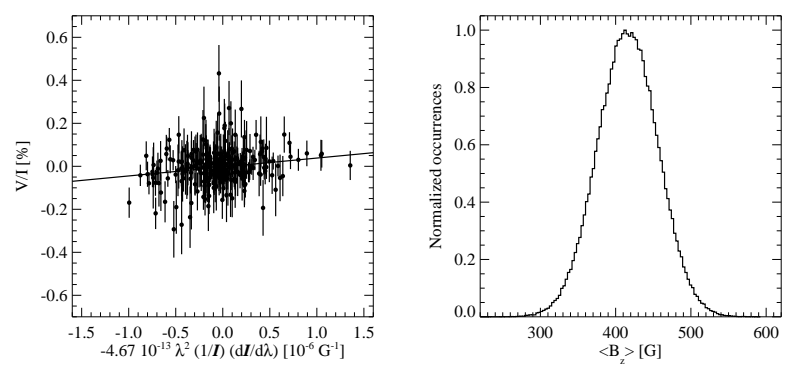

Figure 3. Left panel: Linear fit to Stokes $V$ spectrum obtained for the FORS 2 observation of IGR J11215-5952 on MJD 57738.2863. Right panel: Distribution of the longitudinal magnetic field values $P\left(\left\langle B_{\mathrm{Z}}\right\rangle\right)$, which were obtained via bootstrapping. From this distribution follows the most likely value for the longitudinal magnetic field $\left\langle B_{\mathrm{z}}\right\rangle_{\mathrm{hydr}}=416 \pm 110 \mathrm{G}$.
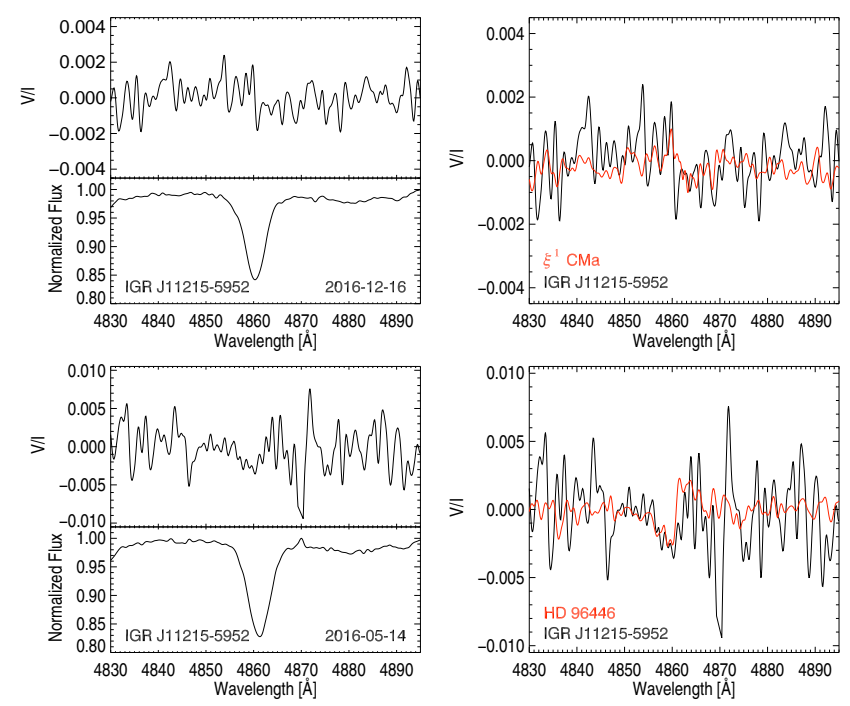

Figure 4. Left panel: Stokes $V$ and Stokes $I$ spectra of IGR J11215-5952 in the spectral region around the $\mathrm{H} \beta$ line at two different epochs. Right panel: Stokes $V$ spectra of IGR J11215-5952 are overplotted with the Stokes $V$ spectra of the two well-known magnetic early-B type stars $\xi^{1} \mathrm{CMa}$ $\left(\left\langle B_{\mathrm{Z}}\right\rangle_{\mathrm{hydr}}=360 \pm 49 \mathrm{G}\right)$ and $\mathrm{HD} 96446\left(\left\langle B_{\mathrm{z}}\right\rangle_{\mathrm{hydr}}=-1590 \pm 74 \mathrm{G}\right)$ for best visibility of the Zeeman features.

of spurious polarization signatures. The slopes of the lines fitted to the data directly translate into the values for $\left\langle B_{z}\right\rangle$. In Fig. 4 we present Stokes $V$ spectra of IGR J11215-5952 obtained on these two nights in the spectral region around the $\mathrm{H} \beta$ line. For best visibility of the Zeeman features, we overplot the Stokes $V$ spectra of IGR J11215-5952 with the Stokes $V$ spectra of the two well-known magnetic early B-type stars HD 96446 and $\xi^{1} \mathrm{CMa}$.

Regarding the significance of the magnetic field detections in massive stars at significance levels around $3 \sigma$, we note that the two clearly magnetic Of?p stars HD 148937 and CPD $-28^{\circ} 5104$ have been for the first time detected as magnetic in our FORS 2 observations at significance levels of $3.1 \sigma$ and $3.2 \sigma$, respectively (Hubrig et al. 2008, 2011). The detection of the magnetic field in IGR J11215-5952 at significance levels of $3.2 \sigma$ and $3.8 \sigma$ indicates that this target likely possesses a $\mathrm{kG}$ magnetic field.

According to the orbital phases presented by Lorenzo et al. (2014), the negative magnetic field $\left\langle B_{\mathrm{z}}\right\rangle_{\mathrm{hydr}}=-978 \pm 308 \mathrm{G}$ is detected at the orbital phase 0.958 while the positive magnetic field 

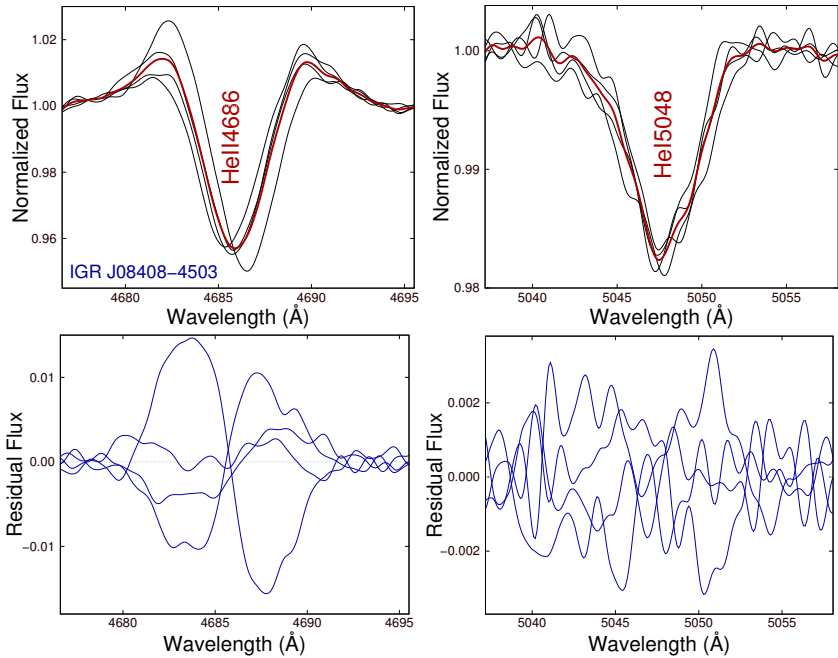

Figure 5. The variability of the He II 4686 line (left side) in the spectra of IGR J08408-4503 on four different orbital phases. The upper and lower panels show the overplotted profiles and the differences between the individual and the average (red) line profiles. No significant variability exceeding the noise level is detected in the He 5048 line (right side)
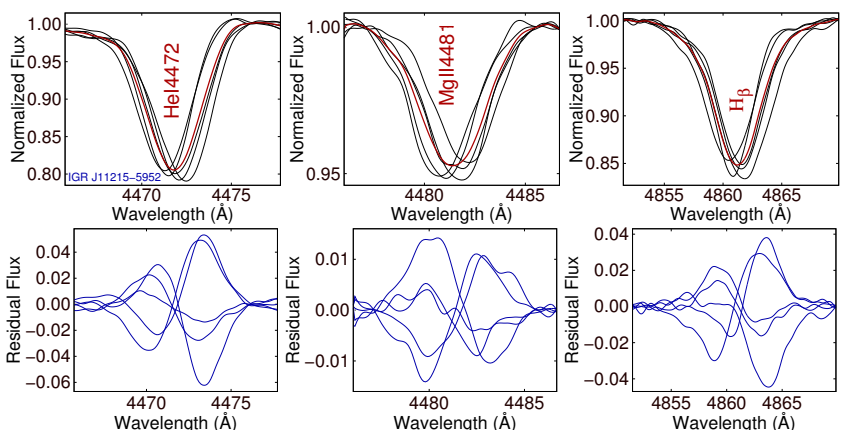

Figure 6. The variability of the spectral lines $\mathrm{He}_{\mathrm{I}} 4472, \mathrm{H} \beta$, and $\mathrm{He}$ I 5048 in IGR J11215-5952 on five different observing nights. The upper and lower panels show the overplotted profiles and the differences between the individual and the average (red) line profiles.

$\left\langle B_{\mathrm{z}}\right\rangle_{\mathrm{hudr}}=416 \pm 110 \mathrm{G}$ is detected at the phase 0.272 . The phase 0 in Lorenzo et al. (2014) corresponds to their first optical observation performed on MJD 54072.33. and has nothing to do with the true periastron passage. Using for IGR J11215-5952 the estimate of the stellar radius $R=40 \pm 5 R_{\odot}$ and $v \sin i=50 \mathrm{~km} \mathrm{~s}^{-1}$ (Lorenzo et al. 2014), the rotation period $P_{\text {rot }}$ is expected to be equal or less than 40.5 d, i.e. its length can be at least four times shorter than the orbital period of $164.6 \mathrm{~d}$. We can speculate that if the ratio between $P_{\text {rot }}$ and $P_{\text {orb }}$ is indeed a factor of four, then the same region of the stellar surface of the optical counterpart of IGR J11215-5952, HD 306414, would face the compact component during the periastron passage.

\section{SPECTRAL VARIABILITY}

Since the presence of pulsations is frequently reported in early type supergiants (see the most recent literature overview presented in the work of Lorenzo et al. 2014), we checked the stability of the line profiles in the FORS 2 spectra of IGR J08408-4503 and IGR J11215-5952 between the nights and along the full sequences

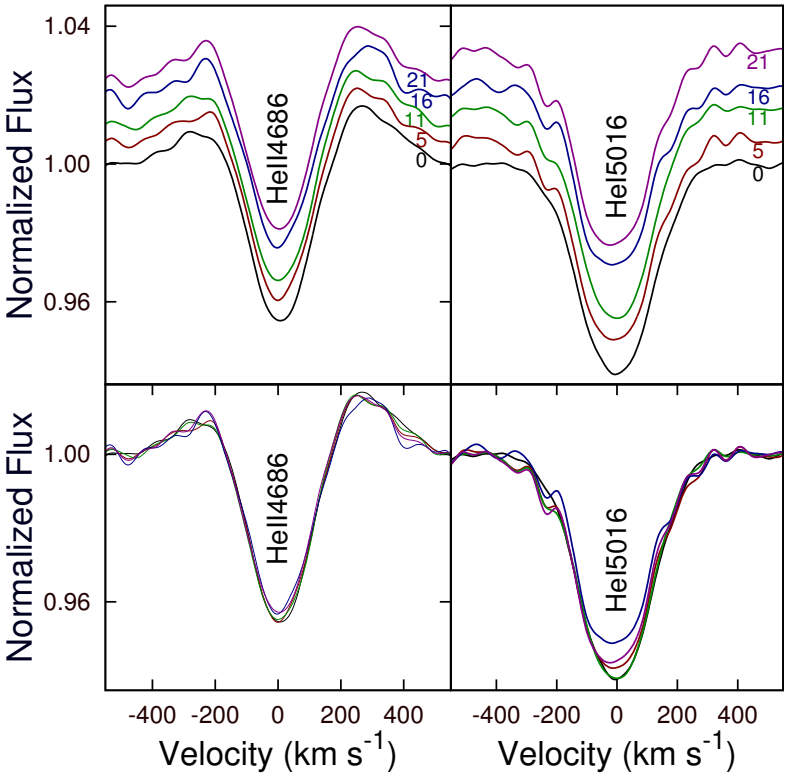

Figure 7. The behaviour of He II 4686 and He I 5016 in the spectra of IGR J08408-4503 obtained for individual subexposures belonging to the observation on 2016 December 5. In the upper row, we present the line profiles shifted in vertical direction for best visibility. The time difference (in minutes) between each subexposure and the start of observations is given close to each profile. The lower row shows all profiles overplotted.

of sub-exposures during each observing night. While only the He II 4686 line was detected as variable in the four FORS 2 spectra of IGR J08408-4503 distributed over 20 days (Fig. 5), most line profiles in the five spectra of IGR J11215-5952 distributed over 271 days show intensity variations and small radial velocity shifts (Fig. 6. As discussed by Lorenzo et al. (2014), the spectral variability of IGR J11215-5952 can be caused by orbital movement combined with pulsational variability. The detected spectral variability can, however, also be caused by a surface inhomogeneous distribution of several elements as is usually observed in magnetic early-type Bp stars. Future careful high-resolution spectroscopic monitoring of this target would be useful to identify the origin of the detected variability.

To search for variability on short time scales, we have compared for each observation the Stokes $I$ line profiles recorded in each subexposure. Only very low intensity variability is detected in IGR J08408-4503 in the He II 4686 line, while it is stronger for the He I lines. In Fig. 7, we present the individual Stokes I profiles of He II 4686 and He I 5015. The line profiles in the FORS 2 spectra of IGR J11215-5952 look identical within the noise.

\section{DISCUSSION}

The formation, evolution, and fate of SFXTs is only partly understood, due to our limited knowledge about the evolution of massive stars. Obviously, understanding these systems is of immense interest, as they probably evolve to NS-NS or NS-BH binaries and are progenitors of gravitational wave signals.

The persistent presence of $\mathrm{H} \alpha$ emission in IGR J08408-4503 can be explained by different physical processes, such as a departure from local thermodynamic equilibrium or/and by light scattering in a geometrically extended atmosphere. On the other hand, it is also possible that the star supports a centrifugal magnetosphere 
with magnetically confined material forming a disk-like structure around the magnetic equatorial plane. Depending on the dipole magnetic field strength and stellar rotation rate, such a magnetosphere would extend to the Alfvén radius of several stellar radii, allowing magnetized clumps of stellar wind to trigger sporadic reconnection during the periastron passage and mass accretion onto the compact companion, which would then result in an X-ray flare. Magnetized clumps caught in the region between the corotating $\mathrm{Ke}$ plerian radius and the Alfvén radius would be centrifugally supported against infall, and so build up to a much denser centrifugal magnetosphere. The non-detection of a magnetic field in our observations of IGR J08408-4503, which are all in orbital phases close to the periastron passage, might be explained by a magnetic field configuration in which we view the magnetic equator of the dipole during these phases. This would also require that the supergiant either rotates synchronously or in resonance with the orbit.

Our spectropolarimetric observations of IGR J11215-5952 revealed the presence of a magnetic field on two occasions. This target is the only SFXT where strictly periodic X-ray outbursts have been observed, repeating every 164.6 d Sidoli et al. 2006, 2007; Romano et al. 2009). To explain these short periodic outbursts, Sidoli et al. (2007) proposed that they are triggered by the passage of the neutron star inside an equatorial enhancement of the outflowing supergiant wind, focussed on a plane inclined with respect to the orbit. This configuration of the line-drive stellar wind might be magnetically channeled (ud-Doula \& Owocki 2002). The effectiveness of the stellar magnetic field in focussing the wind is indicated by the wind magnetic confinement parameter $\eta$ defined as $\eta=B_{*}^{2} R_{*}^{2} /\left(\dot{M}_{\mathrm{w}} v_{\infty}\right)$, where $\mathrm{B}_{*}$ is the strength of the magnetic field at the surface of the supergiant, $\mathrm{R}_{*}$ is the stellar radius $\left(\mathrm{R}_{*}=40 \mathrm{R}_{\odot}\right)$, $v_{\infty}$ is the wind terminal velocity $\left(v_{\infty}=1200 \mathrm{~km} \mathrm{~s}^{-1}\right)$ and $\dot{M}_{\mathrm{w}}$ is the wind mass loss rate $\left(\dot{M}_{\mathrm{w}}=10^{-6} \mathrm{M}_{\odot} \mathrm{yr}^{-1}\right.$; Lorenzo et al. 2014). Adopting $\mathrm{B}_{*} \geqslant 0.7 \mathrm{kG}$ at the magnetic equator, we estimate $\eta \geqslant 500$, implying a wind confinement, up to an Alfvén radius $R_{A}=\eta^{1 / 4} R_{*} \geqslant 4.73 R_{*}$ (ud-Doula \& Owocki 2002). This radial distance is compatible with the orbital separation at periastron in IGR J11215-5952, where the orbital eccentricity is high (e>0.8; Lorenzo et al. 2014). The measured magnetic field strength in IGR J11215-5952 reported here for the first time is high enough to channel the stellar wind on the magnetic equator, supporting the scenario proposed by Sidoli et al. (2007) to explain the short periodic outbursts in this SFXT.

Long-term intensity and radial velocity variability of the wind line $\mathrm{He}_{\mathrm{II}} 4686$ and short-term intensity variability in the $\mathrm{He}_{\mathrm{I}}$ lines were detected in IGR J08408-4503 and can probably be explained by the strong wind in the hot supergiant and by obscuration caused by the surrounding material. The long-term variability intensity and radial velocity variability of the hydrogen and He lines in IGR J11215-5952 was already detected by Lorenzo et al. (2014) and is probably produced by stellar pulsations or surface chemical spots.

Because of the faintness of SFXTs - most of them have a visual magnitude $m_{V} \geqslant 12$, up to $m_{V} \geqslant 31$ (Sidoli 2017; Persi et al. 2015) - no high-resolution spectropolarimetric observations were carried out for them so far and the presented FORS 2 observations are the first to explore the magnetic nature of the optical counterparts. Future spectropolarimetric observations of a representative sample of SFXTs are urgently needed to be able to draw solid conclusions about the role of magnetic fields in the wind accretion process and to constrain the conditions that enable the presence of magnetic fields in massive binary systems.

\section{ACKNOWLEDGMENTS}

Based on observations obtained in the framework of the ESO Prgs. 097.D-0233(A) and 098.D-0185(A). LS acknowledges financial contribution from the grant from PRIN-INAF 2014 "Toward a unified picture of accretion in High Mass X-Ray Binaries". AK acknowledges financial support from RFBR grant 16-02-00604A. We thank the referee Jerzy Madej for his useful comments.

\section{REFERENCES}

Angel J. R. P., Landstreet J. D., 1970, ApJ, 160, L147

Appenzeller I., et al., 1998, The ESO Messenger, 94, 1

Cowperthwaite P. S., et al., 2017, submitted to ApJ, also: arXiv: 1710.05840

Gamen R., et al., 2015, A\&A, 583, L4

Hubrig S., Kurtz D. W., Bagnulo S., Szeifert T., Schöller M., Mathys G., Dziembowski W. A., 2004a, A\&A, 415, 661

Hubrig S., Szeifert T., Schöller M., Mathys G., Kurtz D. W., 2004b, A\&A, 415, 685

Hubrig S., Schöller M., Kholtygin A. F., 2014, MNRAS, 440, L6

Hubrig S., Schöller M., Schnerr R. S., González J. F., Ignace R., Henrichs H., 2008, A\&A, 490, 793

Hubrig S., et al., 2011, A\&A, 528, A151

Liu Q. Z., van Paradijs J., and van den Heuvel E. P. J., 2006, A\&A, 455,1165

Lorenzo J., Negueruela I., Castro N., Norton A. J., Vilardell F., Herrero A., 2014, A\&A, 562, A18

Martinez-Nunez S., et al., 2017, SSRv, 212, 59

Persi P., Fiocchi M., Tapia M., Roth M., Bazzano A., Ubertini P., Parisi, P., 2015, AJ, 150, 21

Romano P., Sidoli L., Cusumano G., Vercellone S., Mangano V., Krimm H. A., 2009, ApJ, 696, 2068

Shakura N., Postnov K., Kochetkova A., Hjalmarsdotter L., 2012, MNRAS, 442, 2325

Shakura N., Postnov K., Sidoli L., Paizis A., 2014, MNRAS, 420, 216

Shakura N. I., Postnov K. A., Kochetkova A. Yu., Hjalmarsdotter L., Sidoli L., Paizis A., 2015. ARep, 59, 645

Shakura N., Postnov K., 2017, to appear in PoS Accretion Processes in Cosmic Sources, September 5-10, 2016, St-Petersburg, also: arXiv:1702.03393

Sidoli L., Paizis A., Mereghetti S., 2006, A\&A, 450, L9

Sidoli L., Romano P., Mereghetti S., Paizis A, Vercellone S., Mangano V., Götz D., 2007, A\&A, 476, 1307

Sidoli L., 2017, submitted to the Proceedings of the "XII Multifrequency Behaviour of High Energy Cosmic Sources Workshop", also: arXiv: 1710.03943

Sota A., et al., 2014, ApJS, 211, 10

Steffen M., Hubrig S., Todt H., Schöller M., Hamann W.-R., Sandin C., Schönberner, D., 2014, A\&A, 570, A88

van den Heuvel E. P. J., Heise, J., 1972, Nature Physical Science, 239,67

ud-Doula A., and Owocki S. P., 2002, ApJ 576, 413 